\title{
Editorial: MILD Combustion: Modelling Challenges, Experimental Configurations, and Diagnostic Tools
}

\author{
Alessandro Parente ${ }^{1 *}$ and Mara de Joannon ${ }^{2 *}$ \\ ${ }^{1}$ Aero-Thermo-Mechanics Laboratory, Ecole Polytechnique de Bruxelles, Université Libre de Bruxelles, Brussels, Belgium, \\ 2Istituto di Scienze e Tecnologie per l'Energia e la Mobilità Sostenibili (STEMS), Consiglio Nazionale delle Ricerche (CNR), Naples, \\ Italy
}

Keywords: computational fluid dynamics, chemical kinetics, colorless combustion, distributed combustion, flameless combustion, MILD combustion, laser diagnostics, turbulence-chemistry-radiation interactions

\section{Editorial on the Research Topic}

MILD Combustion: Modelling Challenges, Experimental Configurations, and Diagnostic Tools

\section{OPEN ACCESS}

Edited and reviewed by: Timothy S. Fisher, University of California, Los Angeles, United States

${ }^{*}$ Correspondence: Mara de Joannon mara.dejoannon@stems.cnr.it Alessandro Parente

alessandro.parente@ulb.be

Specialty section: This article was submitted to Thermal and Mass Transport,

a section of the journal Frontiers in Mechanical Engineering

Received: 17 June 2021 Accepted: 13 August 2021 Published: 04 October 2021

Citation: Parente $A$ and de Joannon M (2021) Editorial: MILD Combustion: Modelling

Challenges, Experimental

Configurations, and Diagnostic Tools.

Front. Mech. Eng 7:726633.

doi: 10.3389/fmech.2021.726633
Over the last years, particular attention has been paid to combustion regimes that are able to ensure stable, complete, and efficient combustion, together with a strong reduction of pollutants, such as $\mathrm{CO}, \mathrm{NO}_{\mathrm{x}}$, and soot. Moderate or Intense Low oxygen Dilution (MILD) combustion (Cavaliere and de Joannon, 2004) has gathered increasing attention in recent years, as it ensures very high combustion efficiencies with very low pollutant emissions, compared to conventional combustion regimes, due to the reduced temperature peaks and macroscale homogeneity, achieved by means of high recirculation of exhausts in the reaction volume. Such a combustion regime shares similarities with other combustion concepts, such as flameless combustion (Wünning and Wünning, 1997), high temperature air combustion (HiTAC) (Katsuki and Hasegawa, 1998), and colorless distributed combustion (CDC) (Arghode and Gupta, 2010). The acronym MILD will be retained for the rest of the present editorial.

MILD combustion has been implemented in several furnace-based power generation and manufacturing applications; however, its extensive application is still partially hindered by the limited understanding of many facets of underpinning elementary processes and their peculiar interplay in this combustion mode.

One of the major peculiarities derived from the change of the elementary processes is related to the very strong interactions between turbulence and chemistry occurring in this regime. In MILD combustion, two contrasting effects emerge: Damköhler number is of the order of unity or less, whereas the turbulence level is very high, to ensure effective gas recirculation and mixing at the microscale. These two characteristics lead to a new paradigm of the combustion process where no flame fronts are visible and the combustion process covers an extensive part of the combustion chamber. It is evident that some principles, well consolidated for a standard combustion process, are no longer applicable in MILD combustion. For example, many common combustion model assumptions, such as the infinitely thin reaction zone, cannot be made in MILD conditions. Analogously, consolidated kinetic models are not able to accurately reproduce experimental data at least in some relevant working conditions.

To overcome these issues, many effective tools for studying and designing MILD combustion processes and systems with novel conceptual approaches have been developed over the years. However, consolidated combustion models and a consistent experimental database are still required to identify MILD combustion regimes and to effectively represent chemical kinetics, heat transfer, turbulence-chemistry interactions, and other processes in this peculiar regime. 
The objective of this research topic is to highlight and discuss open issues, opportunities, and new findings in MILD combustion, with a focus on modelling approaches, experimental configurations (available and under development), and the critical assessment of existing diagnostic tools.

The research topic has two main cores. The first is represented by reviews on key issues faced over the years since the formal definition of MILD combustion in the early 2000s. This part represents an excellent reference summary for an exhaustive overview of this process, with the accent on the open questions.

Indeed, the review by Sabia and de Joannon highlights the critical effects of the high dilution level on the chemical kinetics of fuel oxidation, focusing on the role of diluent species. Based on the literature data, they show how the overall reduction of reaction rates due to dilution stresses the competition among different kinetic paths and brings out very peculiar behaviors, previously undetected, that help better understand chemical kinetics and the role of third body effect in MILD and standard combustion conditions.

$\mathrm{Li}$ and Parente thoroughly review the application of reactorbased models to the simulation of a canonical MILD combustion system, the jet in hot co-flow (JHC). The effectiveness of the partially stirred reactor and eddy dissipation concept combustion models in the context of Reynolds Average Navier-Stokes (RANS) and large eddy simulation (LES) is assessed. The importance of taking into account finite rate chemistry effects and of providing a reliable estimation of the characteristic time scales is underlined.

Reduced reaction rates and the mixing between diluted and/or preheated reactants in MILD combustion locally induce the formation of peculiar reaction structures which are reviewed by Sorrentino et al. They focus on the ignidiffusive structures and their "distributed ignition" nature. The authors analyze the main characteristics of such structures in the mixture fraction space, namely, the thickness of the oxidation structures, the presence/absence of a pyrolysis region, and the loss of correlation between the maximum heat release rate and the stoichiometric mixture fraction.

The peculiar characteristics of such a process also impact the formation of nitrogen oxides (NOx). Iavarone and Parente provide an evaluation of the possible kinetic pathways active in MILD conditions and outline suitable modelling approaches to predict $\mathrm{NO}_{\mathrm{x}}$ emissions in CFD simulations. An assessment of the performances of selected models in estimating $\mathrm{NO}_{\mathrm{x}}$ formation for lab-scale MILD combustion burners is then presented, followed by a discussion about relevant modelling issues, perspectives, and opportunities for future research.

Heat transfer plays a particular role in MILD combustion. Sorrentino et al. highlight the role of heat transfer in the combustion peculiarities of MILD reactors. In particular, the thermal behavior of these systems is analyzed to stress the distinctive role of heat losses, the relative contributions of both the convective and radiative terms, and their influence on MILD macroscopic features.
The experimental study of MILD combustion requires the establishment of new experimental configurations and diagnostic methodologies. Medwell and Evans review a number of optical diagnostic techniques (Rayleigh and Raman scattering, planar laser-induced fluorescence, coherent anti-Stokes Raman scattering ( CARS), and spectroscopy) for the characterization of the MILD combustion of gas and liquid fuels in the JHC.

Chinnici et al. discuss the hybridization of MILD combustion with renewable sources, reviewing the numerical work on a hybrid solar receiver combustor (HSRC), coupling a MILD combustion burner with a concentrated solar radiation receiver. The authors analyze the efficiency of the system as a function of the solar radiation contribution, indicating the requirements in terms of the reactor dimension to reach an appropriate coupling efficiency.

The second core of the research topic is represented by articles focused on original research on different topics under discussion in MILD combustion, covering the fundamental understanding of the process, its numerical modelling, and the identification of optimal reactive scalars to assist experimental diagnostics.

Swaminathan relies on recent direct numerical simulation (DNS) data to show that a revised theory involving at least two chemical timescales is required to describe the inception of MILD combustion and describe the strong interactions between autoignition and flame propagation. Moreover, the relevance of MILD combustion to supersonic combustion is explored theoretically, providing qualitative support using experimental and numerical Schlieren images.

Sidey-Gibbons and Mastorakos analyze the critical phenomena in MILD combustion using an asymptotic theory for extinction conditions of non-premixed flames and well-stirred reactors. Results of the analysis suggest that MILD combustion systems do not show sudden ignition and extinction behavior, and therefore exhibit a smooth, stretched S-shaped curve rather than a folded one with inflection points, thus providing a potential alternative definition of MILD combustion.

Ferrarotti et al. investigate the correlation between the heat releaser rate (HRR) and species mole fractions and net reaction rates in the JHC, suggesting that typical markers $\left(\mathrm{O}, \mathrm{OH}\right.$, and $\left.\mathrm{OH}^{\star}\right)$ correlate fairly well with HRR, but improved correlations can be achieved with appropriate species mole fraction combinations, particularly for the MILD region of the flame.

Goktolga et al. present direct numerical simulations of igniting mixing layers, considered representative of the JHC configuration, using both detailed chemistry and the multistage flamelet-generated manifold (MuSt-FGM) approach. Results indicate that the MuSt-FGM approach can predict the ignition delay time fairly well, while it overpredicts the average heat release rate.

Amaduzzi et al. benchmark the flamelet-generated manifold (FGM) approach with a reactor-based model, the partially stirred reactor (PaSR), for a MILD system with internal recirculation. The results show that the FGM model strongly overpredicts temperature profiles in the reactive region while yielding better results along the central thermocouple. The PaSR closure with a dynamic estimation 
of the mixing constant is found to provide improved results for both lateral and central thermocouple measurements. A flame index analysis indicates how the FGM model predicts a typical non-premixed region after the injection zone, contrary to the experimental observation.

Perpignan et al. present a novel approach for the automatic generation of chemical reactor networks (CRNs) from simplified CFD simulations, for the subsequent evaluation of pollutant emissions. Data from a non-premixed burner fuelled with $\mathrm{CH}_{4}$ at various equivalence ratios are used for this purpose. The CRN results are capable of reproducing the non-monotonic behavior with an equivalence ratio, which cannot be captured by simplified CFD simulations. However, the agreement between experimental and predicted $\mathrm{NO}_{\mathrm{x}}$ emissions is not fully satisfactory, indicating a need for improving the clustering step in the CRN generation process.

In conclusion, the research topic provides a comprehensive overview on the key features and existing challenges in MILD combustion, highlighting the current research efforts and the opportunities ahead. The unique combination of review and

\section{REFERENCES}

Arghode, V. K., and Gupta, A. (2010). Effect of Flow Field for Colorless Distributed Combustion (CDC) for Gas Turbine Combustion. Appl. Energy 87 (5), 1631-1640. Cavaliere, A., and De Joannon, M. (2004). Mild Combustion. Prog. Energy Combust. Sci. 30 (4). Katsuki, M., and Hasegawa, T. (1998). The Science and Technology of Combustion in Highly Preheated Air. Symp. Combust. Proc. 27 (2), 3135-3146.

Wünning, J. A., and Wünning, J. G. (1997). Flameless Oxidation to Reduce Thermal No-Formation. Prog. Energy Combust. Sci. 23 (1), 81-94.

Conflict of Interest: The authors declare that the research was conducted in the absence of any commercial or financial relationships that could be construed as a potential conflict of interest. original research articles makes it a key collection for researchers and practitioners starting or already in the field.

\section{AUTHOR CONTRIBUTIONS}

All authors listed have made a substantial, direct, and intellectual contribution to the work and approved it for publication.

\section{ACKNOWLEDGMENTS}

This Research Topic originated from the first international workshop on MILD combustion that took place in Naples on January 25-26, 2019, with the support of the European Research Council (ERC) under the European Union's Horizon 2020 research and innovation programme under grant agreement no. 714605 (VADEMECOM project, www.vademecom.eu), and the SMARTCATs COST Action CM1404 "Chemistry of Smart Energy Carriers and Technologies" (www.smartcats.eu).

Publisher's Note: All claims expressed in this article are solely those of the authors and do not necessarily represent those of their affiliated organizations, or those of the publisher, the editors, and the reviewers. Any product that may be evaluated in this article, or claim that may be made by its manufacturer, is not guaranteed or endorsed by the publisher.

Copyright (c) 2021 Parente and de Joannon. This is an open-access article distributed under the terms of the Creative Commons Attribution License (CC BY). The use, distribution or reproduction in other forums is permitted, provided the original author(s) and the copyright owner(s) are credited and that the original publication in this journal is cited, in accordance with accepted academic practice. No use, distribution or reproduction is permitted which does not comply with these terms. 